\title{
INFLUENCE OF GRAPH PROPERTIES OF PEER-TO-PEER TOPOLOGIES ON VIDEO STREAMING WITH NETWORK CHANNEL CODING ${ }^{\perp}$
}

\author{
Shirish Karande, Hayder Radha \\ Department of Electrical and Computer Engineering, \\ Michigan State University, \\ East Lansing, MI-48824, USA \\ \{karandes, radha\}@egr.msu.edu
}

\begin{abstract}
Network Channel Coding (NCC) distributes channel coding functions over network nodes participating in common or diverse communication sessions. A particular case of NCC is Network Embedded FEC (NEF) [1]-[2], which has been shown to exhibit significant improvements in the performance of video streaming applications over multicast peer-to-peer (p2p) trees. The placement of NCC/NEF codecs and its utility in improving the throughput performance is in general a function of the underlying $\mathrm{p} 2 \mathrm{p}$ graph topology. In this paper we consider two major forms of $\mathrm{p} 2 \mathrm{p}$ topologies: (1) perfectly structured $k$-ary tree topologies that can be built from (virtually) ideal p2p graphs [3] and (2) unstructured random tree topologies where new nodes randomly join as children to any of the existing peers. The two topologies represent an optimal low diameter structured $\mathrm{p} 2 \mathrm{p}$ topology and a trivial randomly evolving sub-optimal topology, respectively. In this paper, we show the impact of key graph parameters, such as the maximum node-degree $k$ and minimum tree-height $D$, on the performance of NCC in terms of NEF throughput as well as video quality for both structured and unstructured topologies. The utility of NCC/NEF for low-degree and/or less structured $\mathrm{p} 2 \mathrm{p}$ topologies is especially highlighted by demonstrating that, embedding of additional codecs can render the performance of less structured topologies or higher diameter topologies to be almost as good as that of the very well structured low diameter topologies. We also investigate the impact of the graph properties on the placement of the NEF codecs.
\end{abstract}

\section{INTRODUCTION}

In a multi-hop communication, the losses over each hop can accumulate to often render the redundancy in an end-to-end Forward Error Correction (FEC) scheme insufficient to facilitate any data recovery. Within the framework of Network Embedded FEC (NEF) [1]-[2], intermediate nodes in the network are enabled with FEC decoding and encoding functionalities, to facilitate efficient loss recovery within the network so as to avoid the accumulation of the losses. A simplistic and straightforward approach of embedding a NEF codec at every single intermediate node would naturally improve the end-to-end throughput, but that would also increase the processing overhead and the end-to-end latency due to processing/service delays. Thus a salient and important feature of the NEF framework also lies in the fact that
NEF can facilitate throughput improvements without significantly increasing the intermediate processing overhead. This is achieved by avoiding placement of a NEF codec at every node and instead placing them only at a few selected nodes. It has been shown that a near-optimal placement of very few NEF codecs is sufficient to lead to a significant performance improvement in video quality in many network topologies arising from the Transit-Stub Models [3].

Peer-to-Peer $(\mathrm{p} 2 \mathrm{p})$ networks facilitate processing at intermediate nodes and thus provide an opportunity to develop new FEC paradigms such as NEF. However, the Transit-Stub models used for the prior work on NEF [1]-[2] do not appropriately characterize the topology of $\mathrm{p} 2 \mathrm{p}$ networks. It is worth noting that typically $\mathrm{p} 2 \mathrm{p}$ topologies do not contain long (or any) "Transits". Consequently it is essential to evaluate and establish the utility of NEF for typical $\mathrm{p} 2 \mathrm{p}$ topologies, which are represented by graphs with low diameters and closely "packed" nodes/peers. Thus in this paper we analyze the utility of NEF for two p2p topologies: (a) Completely packed trees which are usually obtained from optimal-low diameter p2p topologies which asymptotically approach the Moore Bound [3] (b) A random tree topology which, though representative of $\mathrm{p} 2 \mathrm{p}$ networks, does not have as low a diameter as optimal $\mathrm{p} 2 \mathrm{p}$ topologies and is unstructured (or less structured). Both the above topologies can be characterized by employing a bound on the maximum degree of each node. Details of these topologies and the considered communication networks are provided in section 2 .

In section 3 we investigate the impact of graph properties on the utility and placement of NEF codecs. Section 4 provides results on throughput analysis of the considered topologies, while section 5 provides results based on H.264 video simulations. Results in both sections 4 and 5, clearly illustrate the utility of NEF. Finally we summarize the key conclusions of the proposed work in section 6 .

\section{NETWORK TOPOLOGY}

Perfectly Structured $\boldsymbol{k}$-ary Trees: Optimal low-diameter p2p topologies should asymptotically approach the Moore Bound and thus minimum depth routing trees constructed over such topologies would be appropriately represented by perfectly structured trees. In [3] it was shown that de Bruijn Graphs are near optimal in terms of diameter and routing distances, are most suitable for $\mathrm{p} 2 \mathrm{p}$ networks and get close to achieving the Moore Bound . Thus to represent routing trees emerging from low-diameter well structured $p 2 p$

${ }^{\perp}$ This material is based upon work supported by the National Science Foundation under NSF Theoretical Foundation Award CCF-0515253. 
topologies, we consider a perfectly structured tree topology of $n$ nodes to be such that, node identification (id.) 1 corresponds to the root and any other node with id $m$ is a child of a node with id = $\lfloor\mathrm{m} / \mathrm{max}$. node degree $\rfloor$. Note, this condition is sufficient to determine the entire tree topology. Figure 1 (d), (e), (f) show such trees corresponding to optimal $\mathrm{p} 2 \mathrm{p}$ topologies, for max. node degree $=2,3,4$ and $n=100$. For additional details about graph theoretic analysis of $\mathrm{p} 2 \mathrm{p}$ topologies, please refer to [3] (and references within).

Randomly Evolving Trees: We again consider a tree topology with $n$ nodes, such that node id 1 corresponds to the root/source. The tree topology is generated by a way of a random process in which each node is added to the tree progressively. A node with id $m$ is added to the tree in a random manner, such that, any one of the previously existing $m-1$ nodes is chosen as its parent with uniform probability. We introduce an additional constraint that a node cannot be the parent of more than max. node degree number of children. Figure 1 (a), (b), (c) show examples of such randomly evolved trees for max. node degree $=2,3,4$ and $n=100$. Though such randomly generated trees are not completely unstructured, in this work we address them as unstructured to differentiate them from the more regular/balanced and perfectly structured trees.

Link Behavior: We assume that the losses over each link are memory-less and are governed by a Bernoulli distribution with packet drop probability 0.05 .

\section{CODEC LOCATION}

The codec placement algorithm we use in this paper is identical to the one presented in [2]. Due to brevity, we don't illustrate the algorithm in this document and refer the reader to sections 2 and 3 in [2] for background. In addition, note that all the analysis in this paper is based on the assumption that the FEC coding scheme is based on Reed Solomon (RS) codes, the code/FEC block-length is 30 and number of message symbols/ message packets in each code/FEC block are 24.

Figure 1 shows the location and the order in which 10 NEF codecs are embedded in all of the considered topologies. An important observation to be made is that, the locations of NEF codecs in a

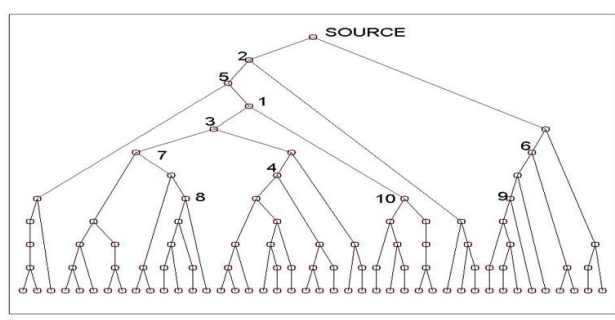

(a) max. node degree $=2$, height $=15$

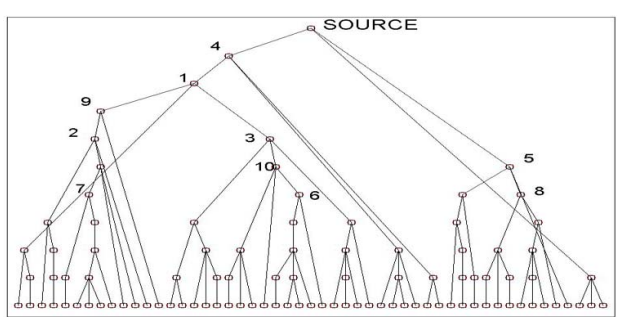

(b) max. node degree $=3$, height $=10$

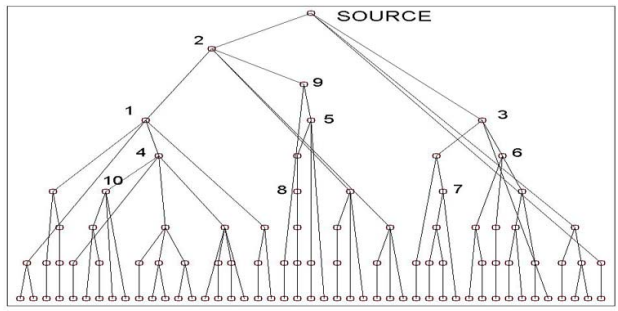

(c) max. node degree $=4$, height $=8$

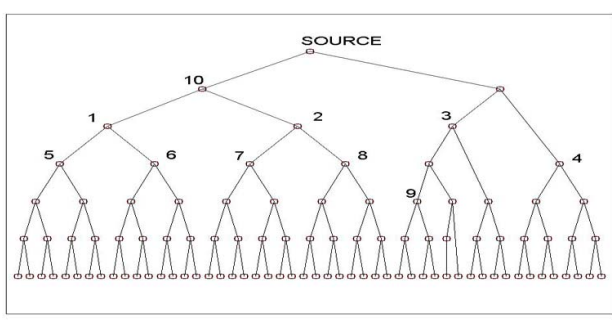

(d ) max. node degree $=2$, height $=6$

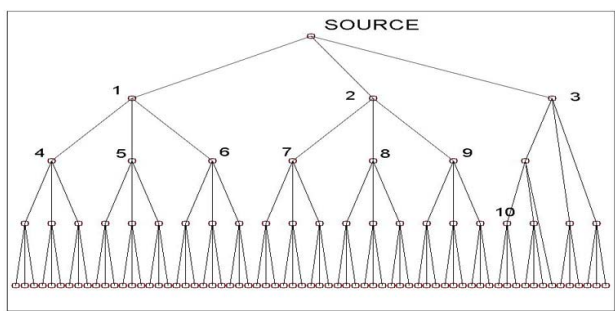

(e ) max. node degree $=3$, height $=4$

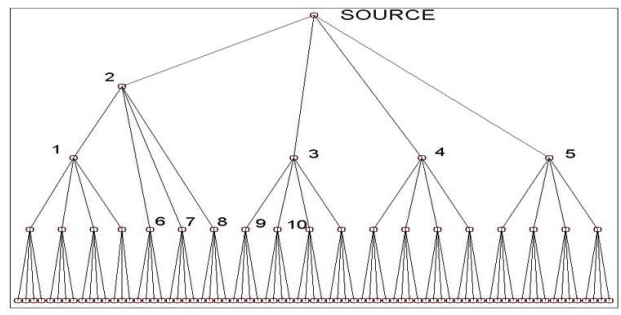

(f ) max. node degree $=4$, height $=4$

Figure 1 Topology of all the considered p2p trees with 100 nodes and the location of the 10 NEF codecs. The codec locations are identified by the order in which they are chosen by the codec placement greedy algorithm. Trees in (a), (b), (c) are obtained by random evolution, while trees (d), (e) (f) are representative of optimal diameter p2p graphs. 
structured graph are also well structured. Thus it may be feasible to develop low time-complexity algorithms that can predict the location of the NEF codecs in structured $p 2 p$ topologies. As against this in a random tree, the placement of the codecs on a cursory inspection appears to lack any pattern/structure. However a closer inspection shows that the codec locations are clustered along a few selected paths. Thus, especially for large networks, reasonable performance improvement could be achieved by mainly concentrating on improving performance along some long/deep paths.

To further investigate the pattern in the location of the NEF codecs, let's for a given tree $T$ consider a set $P_{T}$ of all possible paths from the root node to the leaf nodes. Obviously the number of leafs in a tree vary from one topology to another and thus the number of paths in $P_{T}$ shall also vary. For a path $p \in P_{T}$, let $\sigma(p)$ be the number of NEF codecs on that path. Note that if a path does not have any NEF codec then $\sigma(p)=0$. Finally, let's define:

$$
\begin{gathered}
\text { A concentration coefficient as } \gamma=\underset{p \in P_{T}}{\arg \max } \sigma(p) \text { and } \\
\text { A diversion coefficient as } \delta=\sum_{p \in P_{T}} \sigma(p)
\end{gathered}
$$

The concentration coefficient represents the maximum number of codecs on a single path from the root to a peer and thus represents the worst-case scenario in terms of service delay. Though having many codec's on a single path is not efficient from the perspective of service delay, it may have advantages in terms of managing the tree. The codecs along a single path could be entirely managed by a node other than the source, thus reducing the burden on the root node. The diversion coefficient represents the minimum number of paths (not necessarily vertex/edge disjoint) that have to be considered to span all the codec locations. Thus in some sense the management overhead, i.e. the number of paths that need to be managed is given by the diversion coefficient.

It can be observed that the value of $\gamma$ is $6,5,4,3,2,2$ and the value of $\delta$ is 4, 3, 3, 6, 8, 8 for Figure 1 (a)-(f) respectively. Thus for an identical degree bound, it can be seen that $\gamma$ is always greater for an unstructured random tree, while $\delta$ is always greater for the well structured p2p networks. In addition, as the degree bound is increased, the diameter of the graph is reduced thus leading to shorter paths. Hence, $\gamma$ always reduces as the degree bound increases.

\section{THROUGHPUT IMPROVMENT}

The throughput for each node in all the analysis in this section is defined as

$$
\tau=\frac{\text { No. of message packets recd after FEC decoding by a node }}{\text { No. of message packets trans. by the source node }}
$$

and thus the average throughput for a tree $T$ is given by $E[\tau]$, where the expectation is over all the nodes/peers in the tree.

Thus, it can be observed in Figure 2 that, NEF leads to a significant improvement in average throughput for all the topologies. The improvement is more pronounced for graphs with low degree bound and less structure. For an identical number of peers, degree bound and codecs the performance of a structured

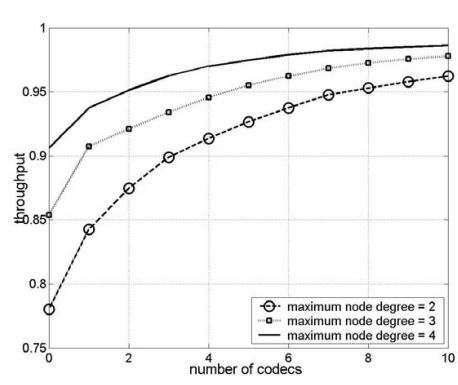

(a)

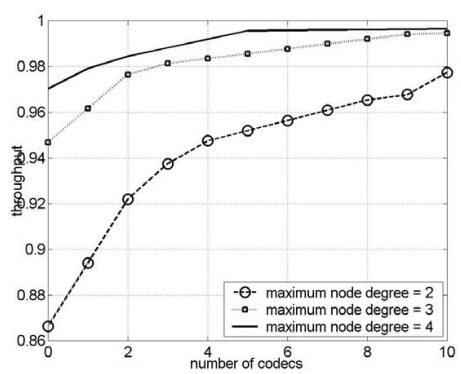

(b)

Figure 2 The improvement in throughput, averaged over all the nodes in the tree, as a function of the number of NEF codec (a) random tree (b) perfectly structured tree

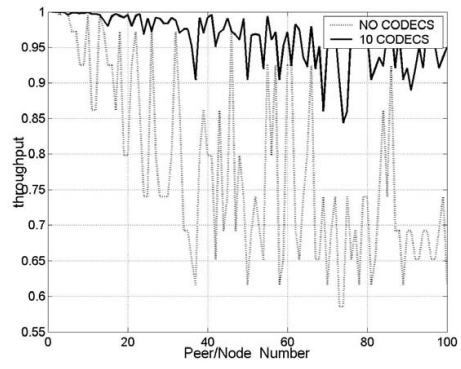

(a)

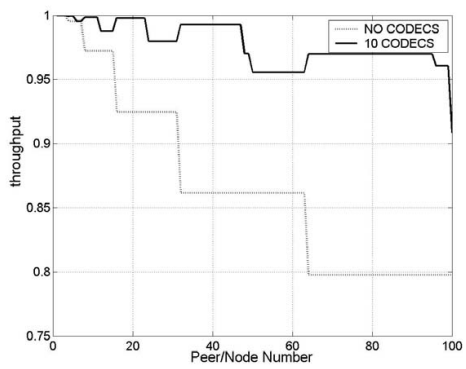

(b)

Figure 3 Comparison between throughputs, of every node in the p2p trees with max. node degree 2 , in presence of $0 \mathrm{v} / \mathrm{s} 10 \mathrm{NEF}$ codecs. (a) random tree (b) perfectly structured tree

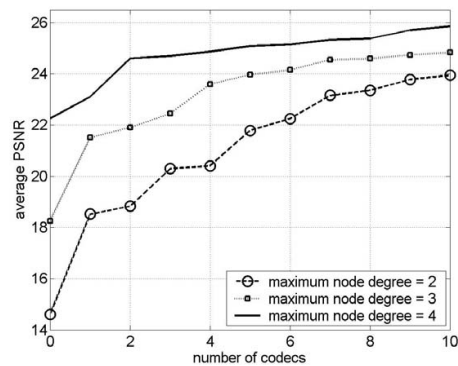

(a)

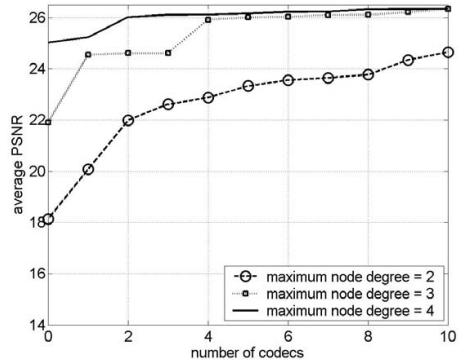

(b)

Figure 4 Video Quality of an "average" node, in terms of PSNR, as a function of the number of NEF codecs in the p2p trees. (a) random tree (b) perfectly structured tree 
p2p topology is always better than a more random one. Similarly for an identical number of peers and codecs, as the degree bound is increased the performance improves. The reason for all the above observation is based on the fact that graphs with low degree bound and less structure have larger depth and thus are more in need of loss recovery at intermediate nodes. However an important observation to be made is that if enough codecs (with the number still being significantly smaller than $n$ and $P_{T}$ ) are embedded, the performance of almost all the topologies can be improved to provide nearly $100 \%$ reliability (Not shown in the figure is the fact that in case of degree bound $=2,15$ codecs are sufficient for throughput to be greater than 99\%). Thus an important consequence of this observation is the fact that, poorly structured $\mathrm{p} 2 \mathrm{p}$ topologies and high diameter topologies can be made to give as good a throughput performance as low-diameter well structured $\mathrm{p} 2 \mathrm{p}$ topologies by just embedding few additional NEF codecs. This fact could be exploited in the design of future $\mathrm{p} 2 \mathrm{p}$ topologies specifically meant for video applications.

In Figure 2, we exhibited the improvement in average throughput; however improvement in average throughput does not provide the complete picture of the utility of NEF. The NEF codec placement algorithm typically places the codec in a location that would be beneficial to the neediest peer/nodes. This facet helps in improving Quality and Service guarantees and thus could play an extremely crucial role in commercial video streaming applications. To illustrate this point, we consider topologies with degree bound 2. Figure 3 shows the value of the throughput $\tau$ for all the peers in such topologies, when no codecs are embedded and when 10 codecs are embedded. It can be seen that the most significant improvement in performance is observed by peers who were receiving the worst throughput when no codecs were embedded.

\section{IMPROVEMENT IN VIDEO QUALITY}

Discussions in the previous section have concentrated on exhibiting the throughput improvements that can be achieved by using NEF. At this stage, it is necessary to clearly establish whether these improvements indeed translate into improvement in the quality of video received by peer nodes. To keep the presentation of the observed results tractable, we present only the performance of the "average" node. The "average" node is represented by the node whose throughput is closest to the throughput averaged over all the nodes in the p2p multicast tree. Thus as the number of NEF codecs embedded in the tree changes, the average throughput changes and thus it's quite feasible that the identity of the "average" node also changes.

The results presented in this section are a subset of the examples we considered and thus it should be stated that the choice of test sequence, quantization, frame frequency and frame size do not compromise on the generalness of the conclusions derived on the basis of the video analysis presented here. However, due to brevity we present results based only on the "stefan" test sequence, a "cif" (352x288) frame size and a frequency of 30 frames/sec. Our simulations are based on a playout of 900 frames obtained by repeating the stefan test sequence three times. We used a constant quantization size of $\mathrm{QP}=28$, a GOP size of 15 frames and a GOP structure of IPPP.... It is also worth noting that the encoded stream is made up of video packets of size 500 bytes each. The reference software version of H.264 used for our simulations was TML 9.0 [5]. The encoding of the sequence was RD-optimized for $30 \%$ losses to increase the error-resilience. The FEC encoding parameters used in this section are identical to those used in the previous section. Thus each FEC packet block consists of 30 packets, out of which 24 are message packets. The size of each packet is fixed at 500 bytes.

Figures 4 (a) and (b) show the improvement in the video quality of the "average node". It can be observed that NEF can improve video quality for all the considered topologies. For well structured and very low diameter topologies the utility of NEF codes is minimal, but even for such topologies it can be observed that addition of a few codecs can ensure nearly loss-less transmission. As the bound on the maximum node degree is decreased, the diameter of the graph and hence the height of the routing tree increases. Thus it can be seen that for high diameter topologies, NEF can provide significant improvement in video quality. In Figure 4(a) it can be observed that for a random topology, for max node degree $=3(2)$ a performance improvement of $6 \mathrm{db}(10 \mathrm{db})$ can be obtained. For the better packed perfectly structured topologies, the performance improvement though less drastic can still be quite significant. In Figure 4(b) it can be seen that when the max-degree is bounded at 3 , embedding just 4 codecs can provide a performance improvement of $4 \mathrm{db}$. If this max-degree bound is reduced to 2, the performance improvement can be as high as $6 \mathrm{db}$. Finally notice that when enough number of codecs are introduced the video quality observed over higher diameter topologies and also less structured topologies is almost as good as the best structured and least diameter topology (for max-node degree 2, 15 codecs were found sufficient to render the PSNR quality to be greater than $25 \mathrm{db}$ ).

\section{CONCLUSION}

It was observed that for the two $\mathrm{p} 2 \mathrm{p}$ topologies considered in this paper, NEF can provide significant gains in throughput and video quality. The performance improvement is more pronounced for topologies with less structure and low bound on the maximum node degree. The utility of NEF for low-degree and/or less structured $\mathrm{p} 2 \mathrm{p}$ topologies was especially highlighted by demonstrating that, embedding of additional codecs can render the performance of less structured topologies or higher diameter topologies to be almost as good as that of the very well structured low diameter topologies. Another important facet demonstrated was the improvement in Quality Guarantees and not just the average throughput.

\section{REFERENCES}

[1] H. Radha and M. Wu, "Overlay and Peer-to-Peer Multimedia Multicast with Network-Embedded FEC," IEEE International Conference on Image Processing (ICIP), October 2004.

[2] M. Wu, S. Karande, and H. Radha, "Network Embedded FEC for Optimum Throughput of Multicast Packet Video," Signal Processing: Image Communication - Special Issue on Video Networking, vol. 20, no. 8, pp. 728-742, September 2005.

[3] D. Loguinov, J. Casas, and X. Wang, "Graph-Theoretic Analysis of Structured Peer-to-Peer Systems: Routing Distances and Fault Resilience," IEEE/ACM Transactions on Networking, vol. 13, no. 5, October 2005.

[4] E.W. Zegura, K. Calvert, and S. Bhattacharjee, "How to Model an Internetwork," IEEE Infocom, 1996.

[5] ISO/IEC JTC 1/SC29/WG11 and ITU-T SG16 Q.6, Draft ITU-T Recommendation and Final Draft International Standard of Joint Video Specification (ITU-T Rec.H.264 | ISO/IEC 14496-10 AVC), Doc.JVTG050, March 2003. 